\title{
Is routine dental prophylaxis effective?
}

\author{
Abstracted from \\ Azarpazhooh A, Main PA. \\ Efficacy of dental prophylaxis (rubber cup) for the prevention of caries and gingivitis: \\ a systematic review of literature. Br Dent J 2009; 207: E14; discussion 328-329 \\ Address for correspondence: Dr Amir Azarpazhooh, Community Dental Health Services Research Unit, \\ and Department of Endodontics, Faculty of Dentistry, University of Toronto, Toronto, Ontario, M5G 1G6, \\ Canada. E-mail: amir.azarpazhooh@dentistry.utoronto.ca
}

\section{Question: Does dental prophylaxis provided at recall appointments reduce caries increments, or improve gingival health?}

Data sources Searches were made for relevant papers using Medline, CINHAL (Cumulative Index to Nursing and Allied Health Literature), Cochrane Central Register of Controlled Trials, Cochrane Database of Systematic reviews, Database of Abstracts of Reviews of Effects, Embase, Health and Psychosocial Instruments, HealthSTAR, International Pharmaceutical Abstracts, and ACP (American College of Physicians) Journal Club. Further articles were identified by reviewing the references and bibliographies of the retrieved articles.

Study selection Articles were limited to original human studies assessing rubber cup dental prophylaxis. All other studies, including in vitro studies, reviews and case series, were excluded. Only studies in English with prophylaxis given at a recall appointment at intervals of 4 months were included.

Data extraction and synthesis The quality of articles was assessed independently and evidence levels rated. A qualitative synthesis is presented.

Results Four articles relating to dental prophylaxis and caries prevention and two articles relating to dental prophylaxis and gingivitis prevention were included. Four studies found that a dental prophylaxis was not warranted before professionally applied topical fluoride (PATF) for caries prevention in children. A generalisation about dental prophylaxis before PATF cannot be applied to adolescents and adults. Available evidence from two other studies fails to demonstrate any benefit in the prevention of gingivitis from further dental prophylaxis at the interval used here for recall examinations. Conclusions To prevent caries in children, dental prophylaxis need not be provided either at a recall visit or before PATF. Dental prophylaxis at intervals of 4 months or more is not justified for the prevention of gingivitis in the general population.

\section{Commentary}

Dental prophylaxis generally consists of mechanical cleaning of the clinical crowns of the teeth, using an abrasive paste and a rubber cup rotating at low speed. This systematic review attempts to assess whether dental prophylaxis provided at recall appointments reduces caries increments, on its own or in combination with PATF, or improve gingival health.

Data for this review was obtained by searching Ovid Medline and several other well recognised databases. The authors made an attempt to include randomised control trials (RCT), although the number of suitable studies of this type was low, and non-RCT were therefore included. There was no personal contact with experts and only papers published in the English language were included, which may have limited the data somewhat.

The authors went into clear detail regarding methods for selecting appropriate studies from the literature search. Further articles were selected by performing a secondary search using the references from the original papers. Following initial exclusions, 12 articles were critically appraised by two separate readers using a checklist to assess evidence of efficacy of therapy or prevention. ${ }^{1}$ Of the articles judged to be acceptable, four related to dental prophylaxis and caries prevention, and two related to dental prophylaxis and gingivitis prevention.

Although checklists are useful in such a review, they have several limitations which should be considered. The main issue involves the scoring system employed, in which equal weighting is given to aspects which carry different levels of importance. For example, the aforementioned checklist gives one point for sufficient duration of a study and the same score for the presence of randomisation. Such a system introduces a great deal of subjectivity to a literature review and should be used with caution. The Cochrane Collaboration explicitly discourages use of scales and checklists in reviews, as evidence shows them to be unreliable tools for assessment of validity. ${ }^{2,3}$

Because of wide variation in the design of the separate studies, no attempt was made to combine the data. Instead, qualitative summaries of each of the studies were provided, with a unanimous indication that there was no significant difference between groups of results in each study. The lack of comparable quantitative data meant there was no opportunity to carry out meta-analysis or sensitivity analysis.

All the papers investigating the relationship between prophylaxis and caries increments used acidulated phosphate fluoride as their PATF of choice. Although this form of fluoride delivery has been shown to be effective in the prevention of caries, it has largely fallen 
out of favour in recent years. Instead, topical fluoride varnishes such as Duraphat $(2.26 \% \mathrm{~F})$ have become more widely used because they can adhere to tooth surfaces and are easier to apply. ${ }^{4,5}$ With this in mind, the relevance of the papers to current clinical practice may be reduced.

An obvious theme running through the review is the lack of control of the subjects' dental care outside the study. This was acknowledged by the authors of this review, and points towards a potentially significant factor in influencing the results. The nature of the studies included means, however, that this would be an extremely difficult issue to resolve.

The overall recommendations made by the review are that:

- for the prevention of caries in children, dental prophylaxis need not be provided either at a recall visit or before the application of topical fluorides; and

- for the prevention of gingivitis in the general population, dental prophylaxis at recall appointments (of intervals of 4 or 6 months) is not effective for the prevention or treatment of gingivitis.

The authors state that in a setting such as Canada, to cease provision of prophylaxis prior to PATF would lead to considerable savings in oral health resources because of the nature of the fee structure. In general dental practice in the United Kingdom, no fee is paid for the provision of prophylaxis in the aforementioned situations and no financial gain would be made on the part of the National Health Service by excluding this treatment.
In general, most of the problems outlined here have been identified by the authors. It is fair to say that the evidence has been overstated, and the recommendations made are based on a limited amount of data. In any case, there appears to be little harm or benefit to the patient either way, whether prophylaxis is provided in these situations or not. Prophylaxis, does, however, have other uses not addressed by these authors: for example, prior to inspection for caries, or in the acclimatisation of an anxious or young patient. Given the limited number of high quality studies and reviews available, further research is clearly needed before any changes in clinical practice can be justified.

\section{Chris Sampson}

Glasgow Dental School and Hospital, University of Glasgow, Glasgow, Scotland, UK

1. Azarpazhooh A, Mayhall JT, Leake JL. Introducing dental students to evidence-based decisions in dental care. J Dent Educ 2008; 72: 87-109.

2. Moher D, Jadad AR, Tugwell P. Assessing the quality of randomised control trials: current issues and future directions. Int I Tech Ass Health Care 1996; 12: 195-208.

3. Jüni $P$, Witschi $A$, Bloch R, Egger M. The hazards of scoring the quality of clinical trials for meta-analysis. J Am Med Assoc 1999; 282: 1054-1060.

4. Beltrán-Aguilar ED, Goldstein JW, Lockwood SA. Fluoride varnishes: a review of their clinical use, cariostatic mechanism, efficacy and safety. J Am Dent Assoc 2000; 131: 589-596.

5. Sorvari R, Meurman JH, Alakuijala P, Frank RM. Effect of fluoride varnish and solution on enamel erosion in vitro. Caries Res 1994; 28: 227-232.

Evidence-Based Dentistry (2010) 11, 16-17. doi:10.1038/sj.ebd.6400704 